\title{
Coronary balloon angioplasty for elderly patients with severe angina
}

\author{
PHILIPPE URBAN, KIM FOX, PETER CREAN, LEONARD SHAPIRO, \\ ANTHONY RICKARDS \\ From the National Heart Hospital, London
}

SUMMARY From February 1983 to December 1986, 65 angioplasty procedures were attempted in 51 patients aged 65 or more. All had angina refractory to medical treatment and $89 \%$ of procedures were performed in patients in Canadian Cardiovascular Society angina class III or IV. Sixty eight per cent of patients had multivessel disease. Angioplasty was attempted on a single vessel in 52 instances $(80 \%)$ and on multiple vessels in $13(20 \%)$. The initial success rate was $75 \%$ for stenosis and $44 \%$ for occlusion. No attempt was made to treat all visible lesions in every case. At least one major complication occurred in $6.2 \%$ of the procedures: $Q$ wave infarction in $4.6 \%$, emergency surgery in $4.6 \%$, and death in $4.6 \%$. After a median follow up of four months (range $1-31) 37 \%$ of the patients in whom the procedure was initially successful were asymptomatic and $76 \%$ were improved by at least one Canadian Cardiovascular Society angina class. Angiographic restenosis occurred in $28 \%$ of treated lesions and $39 \%$ of patients had at least one recurrent stenosis. Among the patients with an initially successful procedure, $11 \%$ needed surgical revascularisation during the follow up period compared with $50 \%$ of those with a failed angioplasty.

Balloon coronary angioplasty can be an effective form of treatment for the relief of severe angina in elderly patients.

Most large studies of transluminal coronary artery balloon angioplasty have been performed in patients with a mean age ranging from 51 to $55 .^{1-3}$ In a rapidly aging population, however, attention should also be directed more specifically towards elderly patients because their response to treatment may differ from that of a younger population. Medical treatment may induce poorly tolerated side effects, and surgical revascularisation in patients over 65 is associated with an increased morbidity and mortality. ${ }^{4}$

We analysed our initial results with coronary balloon angioplasty for 65 consecutive procedures in 51 patients aged 65 or over. We obtained follow up information for both successful and failed procedures, and we evaluated the impact of angioplasty on the need for subsequent coronary artery surgery.

Requests for reprints to Dr Anthony Rickards, The National Heart Hospital, London W1M 8BA.

Accepted for publication 23 June 1987

\section{Patients and methods}

PATIENTS

From February 1983 to December 1986, 51 patients aged 65 or more (median 69 , range 65 to 81 ) underwent coronary balloon angioplasty. Thirteen patients had two procedures during the study period and were included twice, and one patient had three procedures and was included three times (table). The 65 angioplasties that constitute the study group were carried out on $48(74 \%)$ men and $17(26 \%)$ women. Eleven $(17 \%)$ had had previous coronary bypass surgery and $19(29 \%)$ gave a history of previous myocardial infarction. All had angina symptoms that were refractory to maximal tolerated medical treatment and $58(89 \%)$ had severe angina, graded according to the Canadian Cardiovascular Society classification as class III or class IV (recurrent episodes of rest pain). Anginal symptoms were usually long standing with a median duration of 24 months (range 1-264 months). A symptom limited exercise treadmill test was obtained for 44 patients. A median value of stage 1 on the Bruce protocol was 
Table Patient characteristics for 65 consecutive angioplasty procedures in 51 patients aged $\geqslant 65$

\begin{tabular}{lr}
\hline Data & No \\
\hline Number of procedures & 65 \\
Age (median and range) & $69(65-81)$ \\
Sex (number and \% male) & $48(74 \%)$ \\
Previous myocardial infarction & $19(29 \%)$ \\
Previous coronary artery surgery & $11(17 \%)$ \\
Duration of symptoms (median and range, months) & $24(1-264)$ \\
CCS angina class II & $7(11 \%)$ \\
CCS angina class III & $21(32 \%)$ \\
CCS angina class IV + pain at rest & $37(57 \%)$ \\
Single vessel disease. & $21(32 \%)$ \\
Multivessel disease & $44(68 \%)$ \\
Main target lesion: total occlusion & $9(14 \%)$ \\
Main target lesion: stenosis & $56(86 \%)$ \\
\hline
\end{tabular}

CCS, Canadian. Cardiovascular Society

reached (range 0 to 5 ). The test induced chest pain in $40(91 \%)$ and ischaemic electrocardiographic changes in $39(89 \%)$. Severe unstable angina was regarded as a contraindication to exercise testing for 18 patients, and nine of these had transient ischaemic electrocardiographic changes documented at rest.

Diagnostic angiography. was performed separately from angioplasty for all patients. An important stenosis was considered to be present when the estimated reduction in lumen diameter from at least two views was $\geqslant 50 \%$, but angioplasty was only attempted for stenoses $\geqslant 70 \%$. Forty cases $(61 \%)$ had normal systolic left ventricular function or only slight regional hypokinesia and $25(39 \%)$ had moderately or severely impaired left ventricular function. Twenty five $(39 \%)$ had three vessel disease, 19 $(29 \%)$ two vessel disease, and $21(32 \%)$ single vessel disease.

\section{ANGIOPLASTY}

Angioplasty was done by techniques available at the time. For the early cases, non-steerable balloons were used (Schneider Medintag, Zurich, Switzerland), and various steerable systems were later used as they became available (Schneider Medintag; Advanced Cardiovascular Supplies, Mountain View, CA 94099, USA). The femoral approach was used in all cases and the end point was a low residual stenosis assessed angiographically. No attempt was made to treat all visible lesions in every case, and often only the most critical lesion (assessed on the basis of the exercise test and angiograms) was dilated: Nine procedures were undertaken for occluded vessels and 56 for stenoses.

Angioplasty was regarded as a primary success when the residual stenosis was $\leqslant 50 \%$ in the absence of a major complication (emergency surgery within $24 \mathrm{~h}, \mathrm{Q}$. wave infarction; or death). In the case of multivessel angioplasty, athe procedure was considered successful if at least the most critical lesion could be satisfactorily dilated.
Antianginal treatment was given as needed before, during, and after the procedure, but $\beta$ blocking drugs were stopped 24 hours before angioplasty because of their potential $\alpha$ stimulating effect. Intravenous heparin (10000 IU) was given immediately before the procedure, intracoronary nitrates were used during the procedure if spasm was observed, and a calcium channel blocking agent was routinely given to all patients after successful angioplasty until the six month follow up review.

\section{FOLLOW U.P}

Clinical follow up was obtained after a median of four months (range 1-40): for all 46 patients with a successful procedure and for all 16 patients with a failed procedure who lived to be discharged from hospital. Thirty six $(78 \%)$ of the 46 patients with a primary successful procedure underwent follow up angiography after a median of four months (range 1-31), either as part of a routine follow up or because of recurrent or worsening symptoms. Recurrence of the treated lesion was considered to be present when the lumen diameter was reduced by $\geqslant 50 \%$ at the site of the angioplasty.

\section{Results}

\section{INITIAL RESULTS}

Angioplasty was attempted on a total of 91 lesions (1.4/patient) in 79 vessels: the left main stem for two, the left anterior descending for 39, the left circumflex for 12 , the right coronary artery for 24 , and a venous graft for two. The procedure was aimed at a single vessel in $\mathbf{5 2}(\mathbf{8 0} \%)$ patients, at two vessel in 12 $(19 \%)$, and at three in one $(1 \%)$.

Sixty twa $(68 \%)$ of the 91 attempted lesions could be successfully dilated. The primary success rate per procedure was $75 \%$ for stenosis $(42 / 56)$ and $44 \%$ for occlusions (4/9). At least one of the following acute major complications occurred in four $(6.2 \%)$ procedures: emergency. surgery bypass grafting (three patients $(4.6 \%)$ ), new $Q$ wave myocardial infarction (three $(4 \cdot 6 \%)$ ) and death (three $(4 \cdot 6 \%)$ ). The three patients who died all had Canadian Cardiovascular Society class 4 angina with episodes of ischaemic pain at rest and three vessel disease. Two of them had undergone multivessel angioplasty. One patient died of irreversible haemodynamic failure before he could be transported to theatre and the two other patients died despite emergency bypass grafting.

All procedures were followed up after a median of four months (range 1-40 months).

FOLLOW UP FOR SUCCESSFUL PROGEDURES

Seventeen $(37 \%)$ procedures completely relieved angina symptoms and $35(76 \%)$ led to improvement 


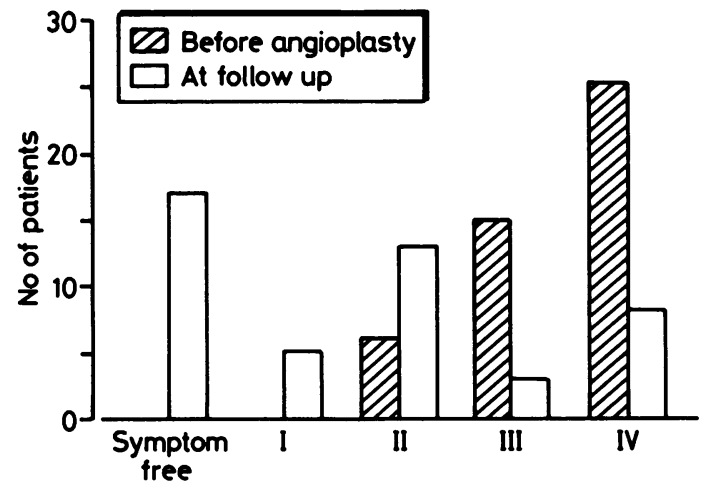

Figure Severity of angina before angioplasty and at follow up (after a median of 4 months, range 1 to 31) for 46 initially successful procedures. Anginal symptoms were graded according to the Canadian Cardiovascular Society; class IV comprises only patients with recurrent episodes of chest pain at rest.

by at least one Canadian Cardiovascular Society angina class (figure). A repeat symptom limited exercise test was available for 31 cases. A median value of stage 2 on the Bruce protocol was reached (range $0-5)$. Eleven (35\%) patients developed chest pain and $10(32 \%)$ had electrocardiographic evidence of ischaemia. Two patients had unstable angina with transient resting electrocardiographic changes at the time of follow up. Twenty nine patients with objective electrocardiographic evidence of ischaemia at rest or during exercise before angioplasty underwent similar evaluation at follow up: the exercise tolerance was improved by one Bruce protocol stage or more for 17 patients $(58 \%)$, remained unchanged for 8 $(28 \%)$ patients, and was decreased for $4(14 \%)$ patients.

For the $36(78 \%)$ cases with follow up angiography, recurrent stenosis occurred in $14(28 \%)$ of the treated lesions and $39 \%$ of cases had at least one recurrent stenosis. A second angioplasty was done with a success rate of $87 \%$ for 14 patients a median of 4 months (range 1-33) after the initial procedure: for restenosis of the same lesion in nine cases, for a different lesion in three cases, and for both in two cases. Elective surgery was done for five $(11 \%)$ patients after a median of seven months (range 1-21). One of the patients died of a non-cardiac cause 14 months after angioplasty.

\section{FOLLOW UP FOR FAILED PROCEDURES}

Follow up data was obtained for all 16 patients in whom procedures failed and who survived to be discharged from hospital. There was one late cardiac death 10 months after attempted angioplasty, and eight $(50 \%)$ patients underwent elective coronary bypass grafting after a median of one month (range 1-40). The frequency of elective bypass surgery during the follow up period after failed angioplasty was significantly higher than after primary successful procedures $(p<0.001)$.

\section{Discussion}

These data confirm previous reports that angioplasty can be effective in controlling angina symptoms in elderly patients and show that this is also true for patients with severe and diffuse coronary artery disease. Our initial success rate was $75 \%$ for stenosis and $44 \%$ for occlusions. At follow up $37 \%$ of the initially successful procedures had completely relieved angina symptoms and $76 \%$ had led to an improvement by at least one angina class.

There is currently no consensus about the influence of age on angioplasty success rate. Timmis et al reported a negative influence of age on initial results, ${ }^{5}$ but this was not confirmed by other investigators. ${ }^{6}$ We found no significant difference when comparing our initial results in the present series with those obtained in 315 procedures in patients under 65 during the same period $(75 \%$ vs $80 \%$ initial success rate respectively).

Mock et al reported on the experience from the National Heart Lung and Blood Institute Registry: 370 patients aged 65 or above underwent angioplasty with an initial success rate of $53 \% .{ }^{7}$ Raizner et al recently reported their experience with angioplasty in an elderly population. ${ }^{6}$ The initial success rate was $81 \%$; at follow up $55 \%$ of patients were symptom free and $91 \%$ were improved. The populations in both these sudies were more selected than the present series with a high prevalence $(65 \%$ and $79 \%$ respectively) of single vessel disease.

Four $(6.2 \%)$ of our patients suffered at least one major acute complication related to angioplasty and three $(4 \cdot 6 \%)$ died during their hospital stay, two of them despite undergoing emergency operation. Although the overall complication rate resembled that in published reports, ${ }^{6-8}$ the mortality rate was high and must reflect the unfavourable combination of three vessel disease together with age and severe angina. In two of the fatal cases multivessel angioplasty had been carried out.

Because of the severity of complications when severe angina was caused by multivessel disease in this age group, we modified our technique and focused our efforts on the most critical lesion without attempting complete revascularisation in every case. This approach has been shown to be effective and probably more safe both in stable and unstable angina. ${ }^{910}$ Twenty one of the 44 patients with multivessel disease were successfully treated with single 
vessel angioplasty. At follow up seven of them needed repeat angioplasty for restenosis, two underwent angioplasty of another lesion, and two were treated for both restenosis and another lesion.

\section{FOLLOW UP}

At follow up $37 \%$ of patients with an initially successful procedure were symptom free and $76 \%$ were improved by at least one angina class. The fact that no more than $37 \%$ of patients were rendered asymptomatic presumably reflects the partial revascularisation; however, grade 1 or 2 angina may not constitute an important limitation for a population with an often sedentary lifestyle. Angiographically, restenosis occurred in $28 \%$ of treated lesions and $39 \%$ of patients had at least one recurrent lesion. Since some patients were studied because of recurrent symptoms and not all patients with an initially successful angioplasty had follow up angiography, it is reasonable to assume that the true frequency of restenosis is somewhat lower. ${ }^{11}$ Previous reports on angioplasty for elderly patients gave little information on the rate of restenosis. Our data suggest that it appears essentially similar to that seen in younger patients. ${ }^{1213}$

The perioperative mortality and the length of hospital stay after elective coronary bypass surgery have been shown to be higher in elderly patients. The mortality was $5 \cdot 2 \%$ for 1086 patients aged 65 or above in the Coronary Artery Surgery Study.$^{4}$ It was thus of interest to note that there was a significant difference in the requirement for elective coronary artery surgery during the follow up period between the patients with an initially successful procedure $(11 \%)$ and those with a failed angioplasty $(50 \%)$. During the perioperative period for the 14 elective operations in this series, one patient developed new $Q$ waves but there were no deaths or cerebrovascular accidents.

\section{Conclusions}

Angioplasty is an attractive therapeutic option for the relief of anginal symptoms in elderly patients, even for those with multivessel disease. It substantially reduces the medium-term need for operation. Acute complications of angioplasty do not seem to be more frequent in older patients, but they are more often fatal. Angioplasty restricted to one vessel per procedure is probably the safest approach for elderly patients with multivessel disease.
$\mathrm{P} \mathbf{U}$ is supported by a grant from the Fonds National Suisse de la Recherche Scientifique.

\section{References}

1 Bredlau CE, Roubin GS, Leimgruber PP, Douglas JS, King SB, Gruentzig AR. In-hospital morbidity and mortality in elective coronary angioplasty. Circulation 1985;72:1044-52.

2 Meier B, Rutishauser W. Transluminal coronary angioplasty. State of the art 1984. Acta Med Scand 1985;701 (suppl):142-7.

3 Sowton E, Timmis AD, Crick JCP, Griffin B, Yates AK, Deverall P. Early results after percutaneous transluminal angioplasty in 400 patients. Br Heart $J$ 1986;56:115-20.

4 Gersh BJ, Kronmal RA, Frye R, et al. Coronary arteriography and coronary artery bypass surgery: morbidity and mortality in patients ages 65 and older. Circulation 1983;67:483-90.

5 Timmis AD, Crick JCP, Sowton E. Factors influencing early results of percutaneous transluminal coronary angioplasty [Abstract]. Br Heart $J$ 1985;53;663.

6 Raizner AE, Hust RG, Lewis JM, Winters WL, Batty JW, Roberts R. Transluminal coronary angioplasty in the elderly. Am J Cardiol 1986;57:29-32.

7 Mock MB, Holmes DR, Vlietstra RE, et al. Percutaneous transluminal coronary angioplasty (PTCA) in the elderly patient: experience in the National Heart, Lung, and Blood Institute PTCA Registry. Am J Cardiol 1984;53:89C-91C.

8 Zaidi AR, Hollman J, Franco I, Simpfendorfer C, Galan K. Coronary angioplasty: can you refer older patients? Geriatrics 1985;40:38-44.

9 Vandoermael MG, Chaitman BR, Ischinger T, et al. Immediate and short-term benefit of multilesion coronary angioplasty: influence of degree of revascularization. J Am Coll Cardiol 1985;6:983-91.

10 de Feyter PJ, Serruys PW, Arnolds A, et al. Coronary angioplasty of the unstable angina related vessel in patients with multivessel disease. Eur Heart $J$ 1986;7:460-7.

11 Blackshear JL, O'Callaghan WG, Califf RM. Medical approaches to prevention of restenosis after coronary angioplasty. J Am Coll Cardiol 1987;9:834-48.

12 Leimgruber PP, Roubin GS, Hollman J, et al. Restenosis after successful coronary angioplasty in patients with single-vessel disease. Circulation 1986;73:710-7.

13 Urban P, Meier B, Finci L, de Bruyne B, Steffenino G, Rutishauser $W$. Coronary wedge pressure: a predictor of restenosis after balloon angioplasty. J Am Coll Cardiol 1987;10 (in press). 